\title{
Quantification of Hand Tissues to Dose Optimization Procedures in Computed Radiology
}

\author{
A.L.M. Pavan ${ }^{1}$, D.R. Pina ${ }^{2}$, G. Giacomini ${ }^{1}$, S. Yamashita ${ }^{2}$, S.M. Ribeiro ${ }^{2}$, \\ S.B. Duarte ${ }^{3}$, and J.R.A. Miranda ${ }^{1}$

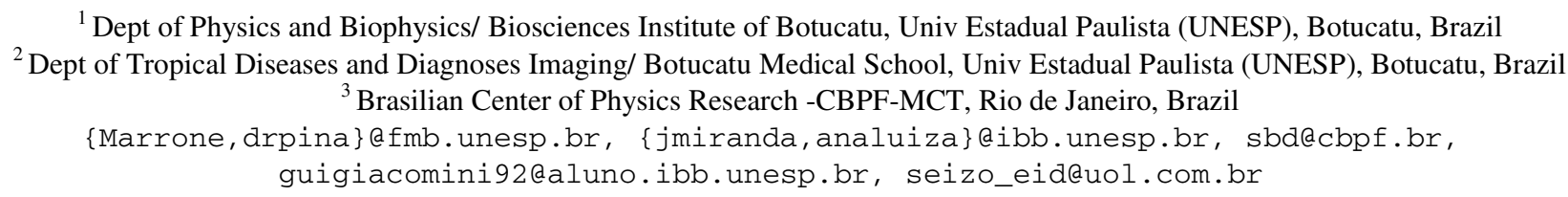

\begin{abstract}
This study presents the development of a homogenous hand patient equivalent phantom (hPEP) to be used in an optimization process in computed radiography systems. Lucite and aluminum plates were used for hPEP construction and their thickness were determined by the equivalence of tissues from anthropomorphic phantom $(2.5 \mathrm{~cm}$ and $0.2 \mathrm{~cm}$ respectively). This phantom was used in $x$-ray beam calibration to obtain the test-techniques of the optimization process with Agfa ${ }^{\circledR}$ exposure index values between 1.8 and 2.0. These test-techniques were applied to an anthropomorphic phantom to the image quality optimization process. In this process the obtained images were assessed by three experienced radiologists through visual grading analysis, and a gold standard (GS) technique was selected on the basis of image quality and As Low As Reasonable Achievable (ALARA) dose. Compared to the previously adopted technique for the clinical routine of the HCFMB-UNESP, the GS technique provided optimal image quality, with reductions in dose and tube load of $41.3 \%$ and $33.2 \%$, respectively. The images generated by using GS technique has satisfactory quality for a safe diagnosis, decreasing the radiation risk to the patient and increasing the lifetime of the $x$-ray tube.
\end{abstract}

Keywords - Optmization, homogeneous phantom, dosimetry, hand, x-ray.

\section{INTRODUCTION}

Homogeneous phantoms are artifacts widely used to radiological quality control and to determine optimal technique and establish protocols for clinical routine. They are constructed employing specific materials with a tissueequivalent amount of material to simulate the absorption and scatter of the x-ray beam in the body [1]. These phantoms are generally made of Polymethyl methacrylate (PMMA-Lucite) and aluminum. Several proposal has been used to simulate different anatomical regions, such as the chest, abdomen, lumbar spine, skull, and extremities [2-5]. However, the development of hand homogeneous phantoms remains unexplored, leaving a lack to optimization of hand images in the radiological literature. Many disease processes are manifested in the small bones of the hands, wrist, and associated soft tissues [6-9]. Several diagnostic decisions depend on the detection of details and the image densities of interfaces in the hand and wrist. Early detection, diagnosis, and continuous evaluation of disease are essential for a successful treatment [10].

We have developed a hand homogeneous patient equivalent phantom (hPEP) in the posterior-anterior projection, which was developed through anthropomorphic phantom's CT examination. The anthropomorphic phantom was the data base to quantification and classification of the different tissues in the hand phantom (epoxy and bone tissue). The estimated epoxy thickness and tissue bone were converted into the corresponding thicknesses of simulator material plates (Lucite and aluminum) in the homogeneous phantom of the hand. The hPEP was used in x-ray beam calibration to obtain the test-techniques of the optimization process. The set of test-techniques was applied to an anthropomorphic phantom, to obtain the gold standard (GS) technique that was associated with a low and safe diagnostic dose, according to the As Low As Reasonable Achievable (ALARA) principle [11]. In this paper, we present an optimized chart of techniques for the adult hand, in a computed radiography (CR) system, to circumvent the need for undesirable and inappropriate trial-and-error procedures currently used in the clinical routine.

\section{MATERIALS AND METHODS}

This study was approved by the Research Ethics Committee of Botucatu Medical School, UNESP, under protocol CEP: 4313-2012

\section{A. Classification and Quantification of hand Tissues}

To the classification and determine the amount of tissues in the hand initially it was used an anthropomorphic phantom, the Sectional Hand Phantoms, XA231R -The Phantom Laboratory, New York, USA. The phantom consisted of correctly positioned genuine bone, cast in epoxy. Epoxy resin simulates soft tissue (fat and muscular tissues) making it possible to assess image quality almost as if it were a real patient [12]. This anthropomorphic hand phantom was sub- 
mitted to tomography scan examination using a SCT7000TS Helical Scanner (Shimadzu Corporation) ), and using the following parameters: pixel size of $0.98 \times 0.98$ $\mathrm{mm}$, matrix of $512 \times 512$ pixels, $120 \mathrm{kV}, 160 \mathrm{~mA}, 1 \mathrm{~mm}$ of increment between slices and $1 \mathrm{~mm}$ of slice width. The whole examination of the anthropomorphic phantom was the data base to quantification and classification the different tissues in the hand. Four types of tissues were considered to be included in the anthropomorphic phantom: fat and muscular tissues, simulated by epoxy material; and trabecular and cortical bone (human skeleton).

As a first step to classify the tissues an experienced radiologist measured the density distribution in HU of the tissues in CT phantom examinations using eleven ROIs of each tissue. The histograms of different ROI's of the same tissue can be fitted by Gaussian type curves or superposition of them [5]. Since fat and cortical bone presents the minimum and maximum densities in the HU range of interest, the corresponding Gaussian's were substituted by a Zfunction and an S-function, respectively. These are named tissues characteristic functions, $\mathrm{Cj}(\mathrm{i})$, and they establish of pertinence of the voxel in the tomography to different tissues in the anthropomorphic phantom. The index $j=0$ is for fat and muscular tissues, represented by epoxy material, $j=1$ for trabecular bones and $\mathrm{j}=2$ for the cortical bone.

The number of voxels of each tissue in the histogram of the anthropomorphic phantom is given by equation 1 . The characteristic function representing the distribution of percentage of each tissue in the histogram, the $\mathrm{Cj}(\mathrm{i})$-functions should be normalized point-to-point by the sum of $\mathrm{Cj}(\mathrm{i})$ functions of the tissues around the i-CT value and then multiplied by the histogram.

$$
\operatorname{Tissue}_{j}(i)=\frac{H(i) C_{j}(i)}{\sum_{j=o}^{2} C_{j}(i)}
$$

To obtain the tissue volume as outputs of this process it is only necessary to multiply the above results by the voxel volume in the CT examination. An important quantity is associated to the tissue volume is the correspondent tissue thickness, define as

$$
\operatorname{Thickness}_{j}(i)=\frac{N_{j} A_{p}}{S(D L L)}
$$

\section{B. The hand Patient Equivalent Phantom (hPEP) development}

With the amount of tissues of the hand evaluated (see table 1) it is possible to determine the mean thicknesses of the anthropomorphic phantom transformed in homogeneous plates, as shows figures $1 \mathrm{a}$ and $1 \mathrm{~b}$, respectevly. The next step was to convert the estimated epoxy thickness and tissue bone into the corresponding thicknesses of the used simulator material plates (Lucite and aluminum) in the homogeneous phantom of the hand. This process was made calculating the amount of thickness of Lucite and aluminum necessary to represent the same attenuation as the original tissues [1]. In this step, quantification values for the composition of biological tissue and epoxy were used.

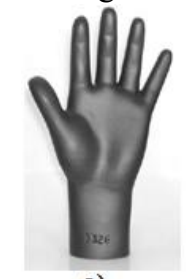

a)

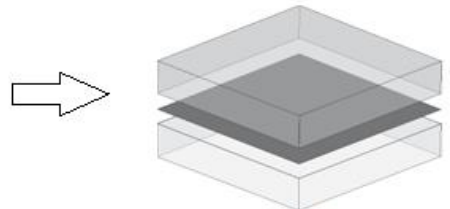

b)
Figure 1. Anthropomorphic (a) and homogeneous (b) phantom.

The hPEP was constructed using aluminum and Lucite plates which is 2 slabs of Lucite of equally thickness with an aluminum foil between them (Gray et al 1983). The dimensions of the plates were: two Lucite slabs of $22.0 \times$ $9.0 \times 1.25 \mathrm{~cm}$ (length $\times$ width $\times$ thickness), simulating epoxy, and an aluminum layer of $22.0 \times 9.0 \times 0.2 \mathrm{~cm}$ between the Lucite slabs, simulating bone tissue.

\section{Optimization of computed Radiography image using hPEP}

The Agfa CR system is used in this work, and the log median exposure level (lgM) index is adopted. The accuracy of the $\operatorname{lgM}$ was tested using AAPM protocol $\# 93$ and the same image plate was used in all x-ray examinations, with a mean interval between exposure and reading of 5 minutes.

According to manufacturer specifications, an appropriated lgM should be around exposure index values between 1.8 and 2.0 [13]. For $x$-ray beam calibration the four mAs values were associated with specific $\mathrm{kVp}$ values between 40 $\mathrm{kVp}$ and $44 \mathrm{kVp}$, with a step of $1 \mathrm{kVp}$, to establish an adequate $\operatorname{lgM}$ index (1.8-2.0). The calibrate procedure generated a set of twenty-one techniques, henceforth called test techniques. These techniques were applied to the anthropomorphic hand phantom, resulting in twenty test images for the hand with $\operatorname{lgM}$ index between 1.8 and 2.0.

Image quality was subjectively assessed by relative visual grading analysis (VGA) [14], and evaluated in a room with dimmed light and surrounding illumination around 30 lux. The VGA assessment of an image can be summarized in a single score through the following equation [15]:

$$
V G A=\frac{\sum O I S_{C}}{N_{I} N_{O}}
$$

